\title{
Role of nasal challenge and local eosinophilia in indirect exposure to cat in allergic rhinitis patients
}

\author{
${ }^{1}$ Microbiology Department, Faculty of Medicine, Kuwait University, Kuwait \\ ${ }^{2} \mathrm{Al}$-Rashed Allergy Center, Ministry of health, Kuwait \\ ${ }^{3}$ Medical Faculty, University of Tuzla, Tuzla, Bosnia and Herzegovina
}

\section{KEY WORDS}

cat; sensitization; nasal challenge;

nasal eosinophil; allergic rhinitis

\section{Corresponding author}

Mona Al-Ahmad

Microbiology Department, Faculty of

Medicine

Kuwait University, P.O. Box 24923

Safat 13110, Kuwait

Phone: +96524636515

Fax: +96525332719

E-mail: Alahmadm@hsc.edu.kw

Doi

10.23822/EurAnnACI.1764-1489.50

\begin{abstract}
Summary
Introduction. Sensitization to cat allergens is common worldwide. Currently, there is a trend towards costly and often unavailable diagnostic analysis. Objectives. The aim is to assess the reliability of skin prick test (SPT) and serum specific IgE (ssIgE) to cat sensitization, by performing nasal challenge test (NCT) in a community with low cat ownership but common presence of stray cats. Patients and methods. Forty-one patients with perennial allergic rhinitis (AR) who were mono or polysensitized (including cat) were included. We had 31 cat non-owners and 10 present cat owners. $S P T$ ( $>5 \mathrm{~mm} /$ diameter), ssIgE ( $\geq 0.70 \mathrm{IU} / \mathrm{ml}$ ), nasal smear for eosinophil (Eo) and NCT were compared between groups. Outcomes included nasal challenge score, nasal Eo positivity, peak inspiratory and expiratory flow (PIF and PEF) 2 and 8 hours after the NCT, and were compared to baseline. Results. Baseline SPT wheal size and ssIgE level were similar in both groups. NCT positivity was more frequent in cat owners. The strongest nasal reaction was on the top concentration in both groups. Nasal Eo positivity in cat owners was higher before and 2 hours after NCT, but similar to non-owners at last measurement. NCT positive cat non-owners had bigger SPT whealsize than NCT negative non-owners, but smaller than NCT positive cat owners. In contrast to PEF, a significant fall in PIF was noticed in both groups. Mono and polysensitised patients showed similar NCT positivity. Conclusion. Stray cats may pose a relevant risk of developing perennial AR. Regardless of cat ownership status, SPT and ssIgE should be the first diagnostic tool. Nasal Eo and NCT seem to be good diagnostic tools in cat non-owners if diagnosis is elusive.
\end{abstract}

\section{Introduction}

Studies have shown that the prevalence of pet sensitization is increasing over the past decades not only in western countries, but also all over the World (1). Together with house dust mites, cat allergens represent the major indoor allergens, and are known risk factors for rhinitis and asthma (2,3). Exposure and sensitization primarily depend on the prevalence of cat ownership, but is still considered ubiquitous (4). The sensitization prevalence varies among countries according to different cultures, climate, environmental factors, traditional and religious believes (5-10). Distribution of the main cat allergens indoors depends on their aero-dynamic diameter and on passive transport by clothes, shoes, or by human hair (11-17) from cat owning home to the environment that has never been occupied by the cat. A study by Woodfolk et al. (18) stressed out the importance of type of vacuum cleaner, which emits cat allergens with a mean of $90 \%$ on particles $<2.5 \mu \mathrm{m} /$ diameter. Similarly, Chapman et al. (19) have documented a wide variation of pet indoor allergens, from less than $1 \mu \mathrm{g}$ to greater than $3000 \mu \mathrm{g} / \mathrm{g}$ of dust, clearly being the highest in pet-owners homes than in non-owners homes.

However, cat ownership should not be considered as the only index of exposure to cat allergens (4). Liccardi had suggested several modalities of exposure to pet allergens and possible con- 
sequences in a "real life" condition, which exclude that any indirect and no apparent contact ( $\mathrm{d}$ and e modalities) should be considered at lower risk of exposure.

Furthermore, Chen et al. (20) documented that exposure to cat allergen concentration as low as $0.24-0.63 \mu \mathrm{g} / \mathrm{g}$ could be positively associated with reported asthmatic respiratory symptoms in subjects who have experienced allergic symptoms when near animals (20). It means that in a community with low cat ownership and common presence of stray cats, the low concentration of cat allergens may be of sufficient magnitude to induce sensitization in susceptible people $(10,19,21)$, and to develop respiratory symptoms after occasional animal contacts (5).

Although cat allergen is the third common allergen in the Middle East countries (22), exposure to cat allergen, sensitization, and its impact on developing allergic rhinitis (AR) and asthma is significant (23). Furthermore, available literature regarding sensitization to stray cats is conflicting (24-27). A study from Kuwait showed that despite low rate of cat ownership (4.1\%) (28), the presence of cat allergens in public buildings is high (29). In contrast to low rate of cat ownership, the sensitization to cat was relatively high (27\%) (30). This was similar to reports from the region $(8,10,22)$. This could be related to numerous stray cats in Kuwait streets and gardens. Therefore, the diagnosis of sensitization to cats is important, irrespective of cat ownership. In up to $95 \%$ cases, the sensitizing allergen to cat (3133 ) is Fel $\mathrm{d} 1$, a glycoprotein which is produced by the sebaceous and salivary glands and transferred to cat fur (34). So cat fur is considered the primary source of cat allergens $(35,36)$. Exposure occurs in public places mostly in countries with high rate of ownership (32,37$39)$, but also in countries with common stray cats $(10,22,29,40)$. Recently there is a trend toward costly component resolved analysis (41-43) instead of standard diagnostic approaches, such as the extract based SPT and serology against native extract (44).

\section{Study design and objectives}

A randomized, controlled, prospective, experimental study was done on allergic rhinitis adult patients with indirect exposure to cat allergen in Al Rashed Allergy Center in Kuwait. The primary objective was to determine diagnostic reliability of SPT wheal size $(\mathrm{mm} /$ diameter) and level of cat serum specific in cat non-owners by performing NCT with cat allergen fur extract. The secondary objective was to determinate the role of NCT. Nasal smear for Eosinophil, PIFR, PEFR, were used as objective measurements of NCT outcome.

\section{Materials and methods}

Forty-one randomly selected adult patients with perennial AR as defined by ARIA guidelines (45) sensitized to cat only or poly-sensitized to cat and at least one more common inhalant allergen, were included and divided into cat owners $(\mathrm{n}=10)$ and cat non-owners $(\mathrm{n}=31)$. Cat owners required a confirmed current direct domestic contact ( $\geq 5$ years). Non-owners never kept cat at home and denied any known direct or indirect exposure to cat. The inclusion criteria included the following: 1, a positive skin prick test with a wheal size of (SPT $\geq 5 \mathrm{~mm}$ ) in diameter, and serum specific IgE $\geq 0.7 \mathrm{IU} / \mathrm{ml}$, to cat only or to cat and at least one common inhalant allergens using a battery of local inhalant allergens (with a long, almost-perennial, pollination); 2, baseline nasal PIFR and PEFR (Clement-Clarke International Ltd, Harlow, Essex UK) within a normal range. The exclusion criteria included: patients with allergic rhinitis and associated asthma.

SPT was performed with a battery of inhalant allergens (Stallergenes, France), including local pollens and cat. Normal saline and histamine were used as negative and positive controls. Skin wheal size (diameter $/ \mathrm{mm}$ ) was recorded after 15 minutes as the mean of 2 perpendicular measurement and was considered as positive as wheal diameter was $\geq 3 \mathrm{~mm}$. SSigE was determined by CAP (Phadia, Pharmacia Sweden).

NCT with cut fur allergen extract (Stallergenes, France) was done at least 3 weeks after acute episode of rhinitis, 1 week after discontinuation of oral antihistamine, nasal cortisone and decongestant, and 2 weeks after antidepressant and oral cortisone $(\geq 10 \mathrm{mg} /$ day). The NCT was performed out of the main pollination peaks following manufacturer recommendation. Frozen dried cat allergen extract $(100 \mathrm{IR} / \mathrm{ml})$, as an active substance, was freshly prepared by reconstitution with $9 \%$ diluent in different concentration starting from 0.1 to maximum $10 \mathrm{IR} / \mathrm{ml}$. After patient's acclimatization ( $\approx 10 \mathrm{~min}$ ) to the physician office condition, the NCT was performed by placing progressive doses of allergen in contact with a patient's nasal mucous membrane, using nebulized cap to deliver $100 \boldsymbol{\mu l} / \mathbf{1}$ puff of allergen solution in each nostril. Nasal reaction was assessed 20 minutes after each dose of allergen, keeping 10 minutes pinched and 10 minutes non-pinched nose as follows: sneezing: $\mathbf{0}=0-2 ; 1=3-4 ; 3=\geq 5$ sneezes; rhinorrhoea and nasal obstruction: $0=$ absence, $1=$ mild, $2=$ moderate; $3=$ severe; nasal palate, eyes and/or ears pruritus: $0=$ absence, $1=$ presence. The test was considered as positive when the total score was $\geq 5$.

Eosinophil nasal smear, as wells as PIFR and PEFR (the best of three measurements was recorded) and compared at three steps: baseline, 2 hours and 8 hours after the challenge.

Nasal samples for Eosinophil positivity were collected by passing a sterile swab, from each nasal cavity, along the medial surface of the inferior turbinate 2 to 3 times, and the specimen smeared on a clear glass slide. Nasal smears were examined by light microscopy after staining with haematoxylin and eosin stain. Eosinophil positivity in nasal smear were calculated in the same time measurement points and compared with a baseline value. Results were interpreted by scale: weak positive ( $4-10 \mathrm{Eo} / \mathrm{hpf})$, moderate (11 - $30 \mathrm{Eo} / \mathrm{hpf}$ ), strong positive (> $30 \mathrm{Eo} / \mathrm{hpf}$ ).

Non-parametric and parametric methods are used to calculate 
statistical significance. The distribution value is determined by D'Agostino and Pearson omnibus test normality. Student's t-test, Mann-Whitney test, Fisher's test and $\chi^{2}$ test were used for calculating the difference between the groups. ANOVA test was used to calculate the relative difference distribution variance between variables. The statistical hypotheses were tested at the level of $\alpha=0.05$, and the difference between the groups in the sample was considered significant when $\mathrm{p}<0.05$ or less. Statistical significance was depicted as: $\mathrm{p}<0.05, \mathrm{p}<0.01$ and $\mathrm{p}<0.001$. All data were analysed using GraphPad Prism version 7 (San Diego, California, USA).

This study was approved by the Ministry of Health ethics approval committee, number $2 / 2016$.

\section{Results}

Cat owners and non-owners showed similar age and gender distribution ( $p>0.05$ for both measurements). The youngest cat owner was 24 years old (female), and the oldest was 54 years old (male). The youngest cat non-owners were 16 years old (male), and the oldest was 57 years old (male).

Mean wheal / diameter (mm) SPT for cat or pollens, as well as ssIgE antibodies level showed similar distribution in cat owners and non-owners ( $p>0.05$ for both measurements).
NCT positivity was more common in cat owners than in cat non-owners $(\mathrm{p}<0.05)$.

Cat owners with positive NCT showed bigger wheal compared to cat non-owners with positive NCT $(\mathrm{p}<0.05)$. However, in cat non-owners, wheal on SPT was bigger in NCT positive in comparison to NCT negative patients $(\mathrm{p}<0.05)$.

PIF showed similar distribution in both groups compared to baseline levels, as well as 2 and 8 hours after NCT ( $p>0.05$ for all measurements). In cat owners, PIF decreased significantly 2 hours after NCT $(\mathrm{p}<0.01)$, but despite recovering 8 hours after NCT it was still lower in comparison to baseline $(\mathrm{p}<0.05)$. However, similarly to cat owners in cat non-owners PIF decreased significantly 2 hours after NCT $(\mathrm{p}<0.0001)$, but 8 hours after NCT PIF recovered completely showing no difference in comparison to baseline $(\mathrm{p}>0.05)$. Meanwhile, PEF remained the same during the challenge in either group ( $p>0.05)$ (table 1).

In both cat-owners and non-owners, most patients reacted on 3 rd concentration $(\mathrm{p}<0.01)$, and then on 2 nd concentration $(p<0.05)$. However, more frequent reactions on top concentration was observed in cat owners compared to non-owners ( $\mathrm{p}$ $<0.01)$. On the other side, similar frequencies were observed among cat-owners and non-owners, on 1st, as well as on 2nd

Table 1 - Patients' baseline and follow up characteristics.

\begin{tabular}{lccc}
\hline Patients & Cat owners & Cat non-owners & p value \\
\hline number of patients & 10 & 31 & \\
\hline age (years) & $37.90 \pm 13.36$ & $31.94 \pm 12.18$ & 0.1959 \\
\hline gender f/m (number) & $8 / 2$ & $12 / 19$ & 0.0564 \\
\hline SPT positive (cat only) & $4(40.0 \%)$ & $12(38.71 \%)$ & 0.7642 \\
\hline SPT cat (mean wheal size in $\mathrm{mm})$ & $9.4 \pm 2.38$ & $8.42 \pm 2.38$ & 0.2673 \\
\hline SPT pollens $(\mathrm{mean}$ wheal/mm) & $6.90 \pm 6.08$ & $7.13 \pm 5.55$ & 0.9122 \\
\hline cat ssIgE $(\mathrm{IU} / \mathrm{ml}$ : mean \pm SD) level & $3[0,5]$ & $3[0,5]$ & 0.9934 \\
\hline positivity of NCT & $10(100 \%)$ & $19(61.29)$ & $0.0179^{1}$ \\
\hline SPT in NCT positive patients $($ mean wheal diameter/mm) & $10.0 \pm 2.75$ & $8.37 \pm 2.06$ & $0.0411^{1}$ \\
\hline SPT in NCT negative patients $(m e a n$ wheal diameter/mm) & - & $7.083 \pm 1.38$ & - \\
\hline p value & - & $0.0118^{1}$ & \\
\hline PIFR before NCT & $67.50 \pm 8.58$ & $65.58 \pm 9.3$ & 0.5669 \\
\hline PIFR 2 hours after NCT & $29.50 \pm 7.98$ & $40.48 \pm 18.04$ & 0.0712 \\
\hline PIFR 8 hours after NCT & $64.00 \pm 7.38$ & $64.32 \pm 7.45$ & 0.9056 \\
\hline p value & $<0.0001^{1}$ & $<0.0001^{1}$ & \\
\hline PEFR before NCT & $469.0 \pm 62.8$ & $459.4 \pm 55.91$ & 0.6476 \\
\hline PEFR 2 hours after NCT & $459.0 \pm 60.64$ & $457.7 \pm 45.51$ & 0.9445 \\
\hline PEFR 8 hours after NCT & $483.0 \pm 51.43$ & $488.1 \pm 51.41$ & 0.7879 \\
\hline p value & 0.6582 & 0.2143 & \\
\hline
\end{tabular}

${ }^{1}$ Difference is statistically significant. 
Figure 1 - Frequency of reactions on different concentration of allergen in NCT in cat owner and cat non-owners.

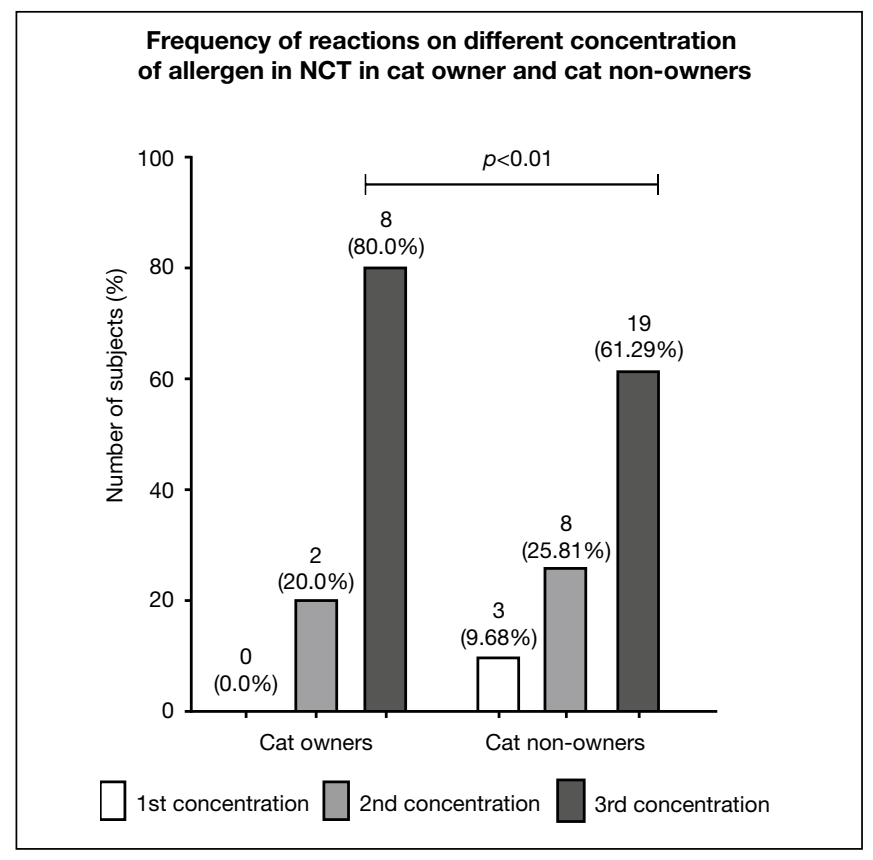

concentration ( $p>0.05$ for both measurements) (figure 1). Cat owners showed similar Eo positivity in nasal smear before NCT, 2 and 8 hours after NCT ( $p>0.05$ ). On the other side, cat non-owners showed more significant Eo positivity in nasal smear 8 hours after NCT compared to positivity before and 2 hours after NCT ( $p<0.01$ for both measurements). Eo positivity in cat non-owners was similar before and 2 hours after NCT ( $p>0.05$ ). However, in comparison to cat non-owners, Eo positivity in cat owners was more pronounced before NCT and 2 hours after NCT ( $p<0.05$ for both measurements), but 8 hours after NCT no significant difference was observed ( $\mathrm{p}>$ 0.05 ) between cat owners and cat non-owners (figure 2).

NCT showed similar positivity between patients sensitised on cat only and patients sensitised on cat and pollen $(p>0.05)$.

\section{Discussion}

In contrast of dog allergy, the role of cat allergens delivered from stray cats in the development of perennial AR is less investigated, both in children and adults. However, the dose of allergen exposure is not always linear (15). So, the relationship between exposure and sensitization to cat allergen is complex and still inconsistent, based on difficulties to classify subjects to direct or indirect allergen exposure (5).

In a study performed in Turkey (10), where cat ownership is low but street cats are common, sensitivity to cat allergen by SPT in adults ranged from $11 \%$ to $44.7 \%$. Our results have shown SPT
Figure 2 - Frequency of Eo positivity of nasal smear before NCT, after NCT and 8 hours after NCT in cat owners and non-owners.

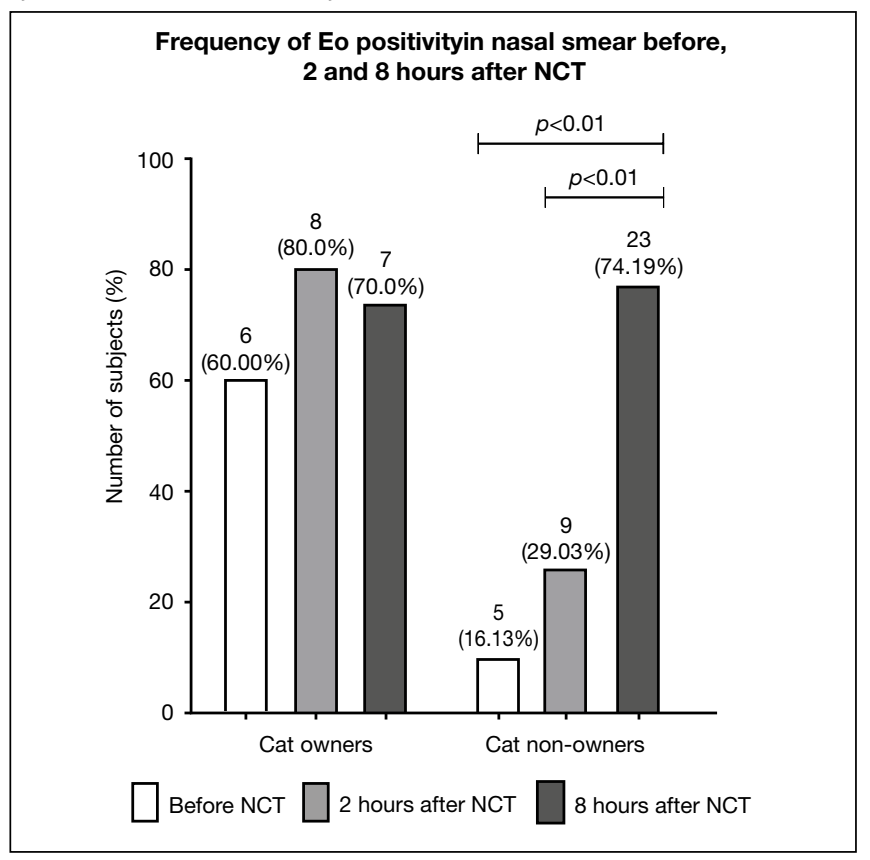

positivity in $40 \%$ of cat owners and $38.71 \%$ of cat non-owners with no significant difference (table 1), which was higher than reported results by others (40).

Furthermore, in 2003 Linneberg A et al. (46) have shown that exposed to cat at home, in adult increased the risk of developing $\mathrm{IgE}$ sensitization to cat.

However, our results have shown similar distribution of ssIgE concentration in cat owners and non-owners (table 1). These findings may point out stray cats as a possible risk factor in the development of perennial AR in adults, regardless on cat owning. On the other side, advanced component based diagnostic testing could not replace SPT and native cat extract serology in the detection of sensitization to cats and differentiation between allergy and sensitization without clinical relevance (47). Complementary with positive ssIgE antibodies, SPT to natural allergen extracts is highly predictive of symptoms development upon allergen exposure $(48,49)$. However, atopy quantification using specific SPT wheal diameter (50) and IgE level may better predict the expression of rhinitis than using atopy as a dichotomous variable (presence / absence of sensitization) (51-53).

NCT may be helpful as additional measurements when the SPT results are not clinically informative enough regarding exposure. However, NCT has not yet been widely accepted in clinical practice (54). Despite of significant difference in NCT positivity in cat owners and non-owners $(100 \%$ vs. $61.29 \%$, p < 0.05 ) in our study, high percentage of NCT positivity in cat 
non-owners could be related to the exposure from stray cuts. We observed bigger SPT wheal size in NCT positive cat owners in comparison to NCT negative cat non-owners $(\mathrm{p}<0.05)$ (table 1), which could be explained by permanent exposure to higher doses of cat allergens in cat owners than in cat non-owners.

Performing a conjunctival challenge with cat allergen extract to determine importance of unnoticed exposure, Braso et al. (55) found positive challenge outcome in 15/20 SPT positive noncat owners with a history of respiratory allergy and exposed to low level (mean of 0.4 microgram/g of dust) of cat allergen. Our results have also shown the bigger SPT wheal size in NCT positive cat non-owners than in NCT negative cat non-owners $(\mathrm{p}<0.05)$ (table 1). All of these subjects had markedly positive SPT ( $>5 \mathrm{~mm} /$ diameter) and ssIgE $\geq 0.70 \mathrm{IU} / \mathrm{ml}$. So, being consistent with literature (55), results from our study support diagnostic importance of wheal SPT size and ssIgE antibodies level. Although (56) Scadding et al. consider NCT as a recognized model that can help to understand the effect of challenging the upper airways on systemic or lower airway inflammation, these authors observed no significant change of PEF during up-dosing in NCT. Also in our study, similar PEF was noticed before, 2 and 8 hours after NCT (table 1) (56). As objective measurement of upper airway obstruction using PIF similar distribution in both group is shown when compared to baseline level, as well as 2 and 8 hours later $(\mathrm{p}>0.05)$. We observed a significant fall in PIF value in the first measurement ( 2 hours) in both groups (cat owners: $p<0.001$; cat non-owners: 0.0001 ) if compared with baseline value. We found recovering after 8 hours, seen by others (56), in non-owner group only ( $p<0.05$ ) (table 1). Our results might be explained with higher sensitivity, but lower specificity of PIF over PEF in detecting of obstruction (57). In the Scadding et al. (56) study conducted on cat owners, significant increase in nasal response between second and highest concentration was absent during NCT. On the contrary, our results have shown that the total nasal score had increased with increasing concentration of cat allergen in both groups $(\mathrm{p}<0.05)$ (figure 1). We speculate that other factors such as life style and climate may influence this phenomenon. The Kuwaiti dwellings, as well as all public buildings, are well ventilated by air conditioning system, which transfer cat allergens indoor. The harsh climate (high temperature, low humidity, and frequent presence of dry dusty wind (58), may increase dispersion and sedimentation of airborne allergens including cat allergen indoors without cats.

Eo in the nasal smear have been reported to display the best correlation with all the clinical and immunological parameters in allergic rhinitis (59). The sensitivity for nasal smear eosinophilia in the diagnosis of allergic rhinitis varies in different studies from $51.3 \%$ to $74 \%$, with a specificity of $88.5 \%$ to $90 \%(60,61)$. However, Eo nasal smears are not necessary for routine use in diagnosing of AR, when the diagnosis is clearly supported by the history, physical examination, SPT and specific IgE diagnostic findings, but may be a useful adjunct when the diagnosis is questionable (62). In our study, Eo positivity in nasal smear was used to evaluate its importance in overall AR diagnostic approach, to evaluate difference between cat owners and non-owners and to estimate local reactivity after NCT as well in both groups. We have shown that cat owners have had significantly higher frequency of Eo positive in nasal smear before NCT, comparing to cat non-owners $(\mathrm{p}<0.05)$. However, cat owners showed similar Eo positivity in nasal smear before NCT and 2 and 8 hours after NCT $(\mathrm{p}>0.05)$. On the other side, cat non-owners showed more significant Eo positivity in nasal smear 8 hours after NCT compared to positivity before and 2 hours after NCT $(p<0.01)$, but in those patients Eo positivity was similar before and 2 hours after NCT $(p>0.05)$. However, Eo positivity in cat owners was more pronounced 2 hours after NCT $(\mathrm{p}<0.05)$ than in cat non-owners, but 8 hours after NCT no significant difference was observed ( $p>0.05$ ) between owners and non-owners (figure 2). Regarding results on increased of Eo in nasal smear in cat owners, it seems that Eo nasal smear could be a helping tool in making a diagnosis of AR. On the other side, in cat non owners, elevated Eo in nasal smear 8 hours after NCT show on possibility of NCT using as decisive tool in making diagnostic of $\mathrm{AR}$ when the diagnose is indeterminate.

The evidence suggests that simultaneous exposure to more than one allergen might modify the effect of individual allergen (15). The same author (24) reported increased prevalence of sensitization to dust mites and pollens in adult pet owners in case of combined exposure. On the contrary, in our study, NCT showed similar positivity between mono (cat) and poly sensitised (cat and pollen) ( $p>0.05)$ (figure 3). Such results suggest that cat allergy could be an independent risk factor for respiratory symptoms in our environment, where prevalence of sensitization to HDM in general is not high (30).

NCT is a safe and helpful procedure in allergy diagnostic. None of patients in either group during up dosing challenge withdrew from further procedure due to clinically significant lower airways symptoms or any other adverse reaction. Similar results are documented by other authors $(54,56,63)$.

In conclusion, in an environment with common presence of stray cats, allergic sensitization to cat without direct exposure may be a relevant risk for developing perennial AR. Regardless of cat owning, SPT wheal size and level of ssIgE $\geq 0.70 \mathrm{IU} / \mathrm{ml}$ should be the first diagnostic tool. NCT and Eo nasal smear seem to be good further method in diagnostic of cat sensitization, especially in cat non-owners.

\section{Patients consent}

All patients were informed about the risk and outcomes of the procedure and provided informed consent. 
Figure 3 - shows positivity of NCT in mono-and poly-sensitised patients.

Positivity of NCT in mono- and polysensitised patients

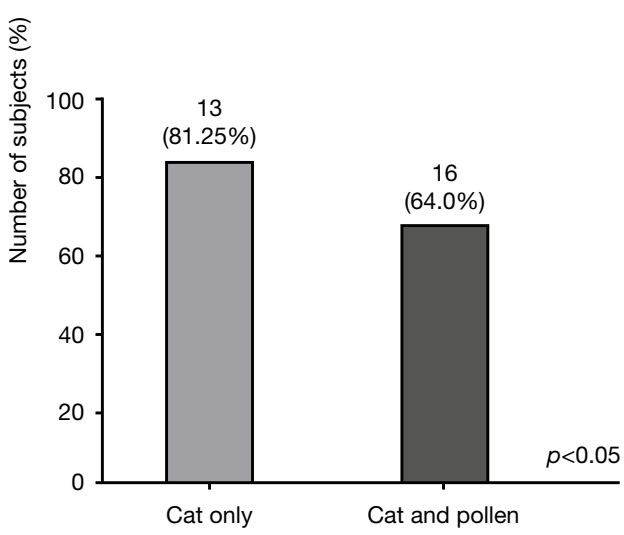

\section{Conflict of interest}

The authors declare that they have no conflict of interest.

\section{References}

1. Katelaris CH, Lee BW, Potter PC, Maspero JF, Cingi C, Lopatin $\mathrm{A}$, et al. Prevalence and diversity of allergic rhinitis in regions of the world beyond Europe and North America. Clin Exp Allergy 2012; 42(2):186-207.

2. Lau S, Illi S, Platts-Mills TA, Riposo D, Nickel R, Gruber C, et al. Longitudinal study on the relationship between cat allergen and endotoxin exposure, sensitization, cat-specific IgG and development of asthma in childhood--report of the German Multicen-tre Allergy Study (MAS 90). Allergy 2005; 60(6):766-73.

3. Perzanowski MS, Ronmark E, Platts-Mills TA, Lundback B. Effect of cat and dog ownership on sensitization and development of asthma among preteenage children. Am J Respir Crit Care Med 2002; 166(5):696-702.

4. Liccardi G, Salzillo A, Calzetta L, Piccolo A, Menna G, Rogliani P. Can the presence of cat/dog at home be considered the only criterion of exposure to cat/dog allergens? A likely underestimated bias in clinical practice and in large epidemiological studies. Eur Ann Allergy Clin Immunol 2016; 48(2):61-4.

5. Liccardi G, Triggiani M, Piccolo A, Salzillo A, Parente R, Manzi F, et al. Sensitization to Common and Uncommon Pets or Other Furry Animals: Which May Be Common Mech-anisms? Transl Med UniSa 2016; 14:9-14.

6. Custovic A, Taggart SC, Woodcock A. House dust mite and cat allergen in different indoor environments. Clin Exp Allergy 1994; 24(12):1164-8.

7. Woodcock A, Addo-Yobo EO, Taggart SC, Craven M, Custovic A. Pet allergen levels in homes in Ghana and the United Kingdom. J Allergy Clin Immunol 2001;108(3):463-5.

8. Farrokhi S, Gheybi MK, Movahed A, Tahmasebi R, Iranpour D, Fatemi A, et al. Common aeroallergens in patients with asthma and allergic rhinitis living in south-western part of Iran: based on skin prick test reactivity. Iran J Allergy Asthma Immu-nol 2015; 14(2):133-8.
9. Stemeseder T, Schweidler B, Doppler P, Klinglmayr E, Moser S, Lueftenegger L, et al. Exposure to Indoor Allergens in Different Residential Settings and Its Influence on IgE Sensitization in a Geographically Confined Austrian Cohort. PLoS One 2017; 12(1):e0168686.

10. Çetinkaya F. BE, Kalayci CO. Cat Allergens in Homes of Cat Allergic Children with-out a Cat. Insights Allergy Asthma Bronchitis 2015; 1:2.

11. Liccardi G, Martin S, Lombardero M, D’Amato M, Barber D, D’Amato $\mathrm{G}$, et al. Cuta-neous and serological responses to cat allergen in adults exposed or not to cats. Respir Med 2005; 99(5):535-44.

12. Almqvist C, Larsson PH, Egmar AC, Hedren M, Malmberg P, Wickman M. School as a risk environment for children allergic to cats and a site for transfer of cat allergen to homes. J Allergy Clin Immunol 1999; 103(6):1012-7.

13. Munir AK, Einarsson R, Dreborg S. Variability of airborne cat allergen, Fel d1, in a public place. Indoor Air 2003; 13(4):353-8.

14. Tranter DC, Wobbema AT, Norlien K, Dorschner DF. Indoor allergens in Minneso-ta schools and child care centers. J Occup Environ Hyg 2009; 6(9):582-91.

15. Custovic A. To what extent is allergen exposure a risk factor for the development of allergic disease? Clin Exp Allergy 2015; 45(1):54-62.

16. Liccardi G, Barber D, Russo M, D’Amato M, D’Amato G. Human hair: an unex-pected source of cat allergen exposure. Int Arch Allergy Immunol 2005; 137(2):141-4.

17. Karlsson AS, Renstrom A. Human hair is a potential source of cat allergen contam-ination of ambient air. Allergy 2005; 60(7):961-4.

18. Woodfolk JA, Luczynska CM, de Blay F, Chapman MD, PlattsMills TA. The effect of vacuum cleaners on the concentration and particle size distribution of airborne cat al-lergen. J Allergy Clin Immunol 1993; 91(4):829-37.

19. Chapman MD, Wood RA. The role and remediation of animal allergens in allergic diseases. J Allergy Clin Immunol 2001; 107(3 Suppl):S414-21.

20. Chen CM, Thiering E, Zock JP, Villani S, Olivieri M, Modig L, et al. Is there a thresh-old concentration of cat allergen exposure on respiratory symptoms in adults? PLoS One 2015; 10(6):e0127457.

21. Lim FL, Hashim Z, Than LT, Md Said S, Hisham Hashim J, Norback D. Asthma, Air-way Symptoms and Rhinitis in Office Workers in Malaysia: Associations with House Dust Mite (HDM) Allergy, Cat Allergy and Levels of House Dust Mite Allergens in Of-fice Dust. PLoS One 2015; 10(4):e0124905.

22. Hasnain SM, Al-Frayh AR, Subiza JL, Fernandez-Caldas E, Casanovas M, Geith T, et al. Sensitization to indigenous pollen and molds and other outdoor and indoor aller-gens in allergic patients from saudi arabia, United arab emirates, and Sudan. World Allergy Organ J 2012; 5(6):59-65.

23. Abdulrahman H, Hadi U, Tarraf H, Gharagozlou M, Kamel M, Soliman A, et al. Na-sal allergies in the Middle Eastern population: results from the "Allergies in Middle East Survey". Am J Rhinol Allergy 2012; 26 Suppl 1:S3-23.

24. Custovic A, Hallam CL, Simpson BM, Craven M, Simpson A, Woodcock A. De-creased prevalence of sensitization to cats with high exposure to cat allergen. J Allergy Clin Immunol 2001; 108(4):537-9.

25. Platts-Mills T, Vaughan J, Squillace S, Woodfolk J, Sporik R. Sensitisation, asthma, and a modified Th2 response in children exposed to cat allergen: a population-based cross-sectional study. Lancet 2001; 357(9258):752-6.

26. Colli Lista G, Perez-Frias J. Exposure to pets and risk of sensitisation to house dust mite, cat and dog allergens in a pre-school children 
population of an Andalusian sea-side town. Allergol Immunopathol (Madr) 2015; 43(6):617-9.

27. Kelly LA, Erwin EA, Platts-Mills TA. The indoor air and asthma: the role of cat al-lergens. Curr Opin Pulm Med 2012; 18(1):29-34.

28. Al-Mousawi MS, Lovel H, Behbehani N, Arifhodzic N, Woodcock A, Custovic A. Asthma and sensitization in a community with low indoor allergen levels and low pet-keeping frequency. J Allergy Clin Immunol 2004; 114(6):1389-94.

29. al Mousawi M, Behbehani N, Arifhodzic N, Lovel H, Woodcock A, Custovic A. Envi-ronmental allergens in Kuwait. Allergy 2001; 56(12):1237-8.

30. Al-Dowaisan A, Fakim N, Khan MR, Arifhodzic N, Panicker R, Hanoon A, et al. Salsola pollen as a predominant cause of respiratory allergies in Kuwait. Ann Allergy Asthma Immunol 2004; 92(2):262-7.

31. Gronlund H, Adedoyin J, Reininger R, Varga EM, Zach M, Fredriksson $\mathrm{M}$, et al. Higher immunoglobulin $\mathrm{E}$ antibody levels to recombinant Fel d 1 in cat-allergic chil-dren with asthma compared with rhinoconjunctivitis. Clin Exp Allergy 2008; 38(8):1275-81.

32. Ichikawa K, Iwasaki E, Baba M, Chapman MD. High prevalence of sensitization to cat allergen among Japanese children with asthma, living without cats. Clin Exp Aller-gy 1999; 29(6):754-61.

33. van Ree R, van Leeuwen WA, Bulder I, Bond J, Aalberse RC. Purified natural and recombinant Fel $\mathrm{d} 1$ and cat albumin in in vitro diagnostics for cat allergy. J Allergy Clin Immunol 1999; 104(6):1223-30.

34. Zahradnik E, Raulf M. Animal allergens and their presence in the environment. Front Immunol 2014; 5:76.

35. Charpin C, Mata P, Charpin D, Lavaut MN, Allasia C, Vervloet D. Fel d I allergen distribution in cat fur and skin. J Allergy Clin Immunol 1991; 88(1):77-82.

36. Portnoy J, Kennedy K, Sublett J, Phipatanakul W, Matsui E, Barnes $\mathrm{C}$, et al. Envi-ronmental assessment and exposure control: a practice parameter--furry animals. Ann Allergy Asthma Immunol 2012; 108(4):223 e1-15.

37. Munir AK, Einarsson R, Schou C, Dreborg SK. Allergens in school dust. I. The amount of the major cat (Fel d I) and $\operatorname{dog}(\mathrm{Can} \mathrm{fI})$ allergens in dust from Swedish schools is high enough to probably cause perennial symptoms in most children with asthma who are sensitized to cat and dog. J Allergy Clin Immunol 1993; 91(5):1067-74.

38. Lok SD, Davis BE, Cockcroft DW. Prevalence of allergen sensitization in 1000 adults in Saskatchewan. Allergy Asthma Clin Immunol 2017; 13:9.

39. Heinrich J, Bedada GB, Zock JP, Chinn S, Norback D, Olivieri M, et al. Cat allergen level: its determinants and relationship to specific IgE to cat across European centers. J Allergy Clin Immunol 2006; 118(3):674-81.

40. Gulbahar O, Sin A, Mete N, Kokuludag A, Kirmaz C, Sebik F. Sensitization to cat al-lergens in non-cat owner patients with respiratory allergy. Ann Allergy Asthma Im-munol 2003; 90(6):635-9.

41. Borres MP, Ebisawa M, Eigenmann PA. Use of allergen components begins a new era in pediatric allergology. Pediatr Allergy Immunol 2011; 22(5):454-61.

42. Canonica GW, Ansotegui IJ, Pawankar R, Schmid-Grendelmeier P, van Hage M, Baena-Cagnani CE, et al. A WAO - ARIA - GA(2) LEN consensus document on molecu-lar-based allergy diagnostics. World Allergy Organ J 2013; 6(1):17.

43. Konradsen JR, Fujisawa T, van Hage M, Hedlin G, Hilger C, Kleine-Tebbe J, et al. Allergy to furry animals: New insights, diagnostic approaches, and challenges. J Aller-gy Clin Immunol 2015; 135(3):616-25.

44. Klimek L, Hoffmann HJ, Renz H, Demoly P, Werfel T, Matricardi $\mathrm{PM}$, et al. Diagnos-tic test allergens used for in vivo diagnosis of allergic diseases are at risk: a European Perspective. Allergy 2015; 70(10):1329-31.

45. Brozek JL, Bousquet J, Agache I, Agarwal A, Bachert C, Bosnic-Anticevich S, et al. Allergic Rhinitis and its Impact on Asthma (ARIA) guidelines-2016 revision. J Allergy Clin Immunol 2017.

46. Linneberg A, Nielsen NH, Madsen F, Frolund L, Dirksen A, Jorgensen T. Pets in the home and the development of pet allergy in adulthood. The Copenhagen Allergy Study. Allergy 2003; 58(1):21-6.

47. Eder K, Becker S, San Nicolo M, Berghaus A, Groger M. Usefulness of component resolved analysis of cat allergy in routine clinical practice. Allergy Asthma Clin Immu-nol 2016; 12:58.

48. Heinzerling L, Mari A, Bergmann KC, Bresciani M, Burbach G, Darsow U, et al. The skin prick test - European standards. Clin Transl Allergy 2013; 3(1):3.

49. Wood RA, Phipatanakul W, Hamilton RG, Eggleston PA. A comparison of skin prick tests, intradermal skin tests, and RASTs in the diagnosis of cat allergy. J Allergy Clin Immunol 1999; 103 (5 Pt 1):773-9.

50. Haahtela T, Burbach GJ, Bachert C, Bindslev-Jensen C, Bonini S, Bousquet J, et al. Clinical relevance is associated with allergen-specific wheal size in skin prick testing. Clin Exp Allergy 2014; 44(3):407-16.

51. Marinho S, Simpson A, Marsden P, Smith JA, Custovic A. Quantification of atopy, lung function and airway hypersensitivity in adults. Clin Transl Allergy 2011; 1(1):16.

52. Custovic A, Simpson A. The role of inhalant allergens in allergic airways disease. J Investig Allergol Clin Immunol 2012; 22(6):393-401.

53. Olivieri M, Heinrich J, Schlunssen V, Anto JM, Forsberg B, Janson C, et al. The risk of respiratory symptoms on allergen exposure increases with increasing specific IgE levels. Allergy 2016; 71(6):859-68.

54. Gosepath J, Amedee RG, Mann WJ. Nasal provocation testing as an international standard for evaluation of allergic and nonallergic rhinitis. Laryngoscope 2005; 115(3):512-6.

55. Braso-Aznar JV, Pelaez-Hernandez A, Rochina-Puchades A, Morales-Rubio C, Burches Baixauli E. Etiologic role of unapparent exposure in cat allergy. Allergy 1995; 50(5):447-50.

56. Scadding GW, Eifan A, Penagos M, Dumitru A, Switzer A, McMahon $\mathrm{O}$, et al. Local and systemic effects of cat allergen nasal provocation. Clin Exp Allergy 2015; 45(3):613-23.

57. Grabowski M, Barg W, Chmielowicz B, Kopec A, Panaszek B. [The variability of peak inspiratory flow (PIF) on valves imitating dry powder inhalers resistance during asthma and COPD exacerbations]. Pneumonol Alergol Pol 2007; 75(4):324-30.

58. Sabbah I. AN, Al Ahmed M., Al Enizi A., Al-Haddad A, Al-Ajmi N. Influence of Air Quality Conditions on Asthmatic Patient Visits in Kuwait. J Allergy Ther 2014; 5:6.

59. Gelardi M, Incorvaia C, Passalacqua G, Quaranta N, Frati F. The classification of al-lergic rhinitis and its cytological correlate. Allergy 2011; 66(12):1624-5.

60. Ahmadiafshar A, Taghiloo D, Esmailzadeh A, Falakaflaki B. Nasal eosinophilia as a marker for allergic rhinitis: a controlled study of 50 patients. Ear Nose Throat J 2012; 91(3):122-4.

61. Bakhshaee M. FM, Farzadnia M, Varasteh AR. The Nasal Smear for Eosinophils, Its Value, and Its Relation to Nasal Mucosal Eosinophilia in Allergic Rhinitis Iranian Jour-nal of Otorhinolaryngology 2010; 22:73-8.

62. Wallace DV, Dykewicz MS, Bernstein DI, Blessing-Moore J, Cox L, Khan DA, et al. The diagnosis and management of rhinitis: an updated practice parameter. J Allergy Clin Immunol 2008; 122(2 Suppl):S1-84.

63. Krzych-Falta E, Piekarska B, Sybilski A, Wojas O, Samolinski B. The Safety of Nasal Allergen Challenge Test Assessed in Lower Airways. Iran J Allergy Asthma Immunol 2015; 14(6):581-8. 